\title{
An Age-structured Mathematical Model for the Within Host Dynamics of Malaria and the Immune System
}

\author{
J. Tumwiine • S. Luckhaus • \\ J. Y. T. Mugisha $\cdot$ L. S. Luboobi
}

Received: 26 April 2007 / Accepted: 20 October 2007 /

Published online: 22 November 2007

(C) Springer Science + Business Media B.V. 2007

\begin{abstract}
In the paper, we use a mathematical model to study the population dynamics of replicating malaria parasites and their interaction with the immune cells within a human host. The model is formulated as a system of age-structured partial differential equations that are then integrated over age to obtain a system of nonlinear delay differential equations. Our model incorporates an intracellular time delay between the infection of the red blood cells by the merozoites that grow and replicate within the infected cells to produce new merozoites. The infected red blood cells burst approximately every $48 \mathrm{~h}$ releasing daughter parasites to renew the cycle. The dynamical processes of the parasites within the human host are subjected to pressures exerted by the human immunological responses. The system is then solved using a first-order, finite difference method to give a discrete system. Numerical simulations carried out to illustrate stability of the system reveal that the populations undergo damped oscillations that stabilise to steady states.
\end{abstract}

Keywords Age-structured · Immune system • Parasite replication • Time delay

Mathematics Subject Classifications (2000) 92B05 • 92D25 • 92D30 • 92C37

J. Tumwiine

Department of Mathematics, Mbarara University of Science and Technology,

P.O. Box 1410, Mbarara, Uganda

S. Luckhaus

Max Planck Institute for Mathematics in the Sciences,

Inselstraße 22, 04103 Leipzig, Germany

J. Y. T. Mugisha $(\varangle) \cdot$ L. S. Luboobi

Department of Mathematics, Makerere University,

P.O. Box 7062, Kampala, Uganda

e-mail: jytmugisha@math.mak.ac.ug 


\section{Introduction}

The immune system is an important part of the host defense and consists of innate and adaptive responses that help limit the adverse effects of infection. Innate immune responses are the first line of defense against the pathogens, and have an important role in malaria. The innate responses are essential to limit the initial phase of parasite replication, controlling the first wave of parasitemia and allowing the host time to develop specific adaptive responses that will enable the infection to be cleared. The effective adaptive immune response comprised of two pathways; antibody mediated immunity and cell mediated immunity. These come into play at different stages of the parasite attack. The antibody-mediated immunity blocks parasite invasion of the red blood cells, promotes the antibody dependent immunity cytotoxicity of parasitized red blood cells and blocks the activity of parasite toxins. The cell mediated immunity reduces the blood parasitaemia. The immune system responds to the presence of the malaria parasites by producing more immune effectors that act by reducing the parasite numbers. However, the malaria parasite also uses the parasite protein to stimulate the immune system for production of antibodies which protect the parasite against attack by the immune system. This complexity of the immune system, where several cell types of the host immune system and different parasite stages are involved, strongly suggests the use of mathematical modelling to gain new insights.

Appropriate mathematical models for the dynamics of immune response to the developmental stages of the malaria parasite if well understood can contribute to the design of control strategies of the disease. They can be helpful to answer biologically important questions concerned with pathogenesis, the dynamics of immune response and effectiveness of drug treatment. The earliest model of Anderson [1] involves four populations: uninfected and infected red blood cells, free merozoites and the immune effector responses, coupled as a system of a nonlinear ordinary differential equations that represent the basic processes of growth, stimulation and clearance. From the model results, they conclude that the immune response against merozoites is less effective than the immune response against infected red blood cells, and that therefore vaccines based on infected red blood cells antigens are likely to be more effective than vaccines based on merozoite antigens. This is an important intervention because the rupture of red blood cells release daughter parasites that quickly invade fresh red blood cells to renew the cycle. The red blood cells cycle maintains infection and directly generates disease symptoms. However, the Anderson [1] model ignores the intra-cellular time lag during which development into blood stage parasites occurs.

The Anderson [1] model has been extended by other authors [see 2, 4-6, 9-11] in many directions to understand the immune response to persistent malaria parasites. Three changes introduced in the original model are: gametocyte stage, a background birth/replication rate of the immune cells and two clones of the malaria parasite. These clones are inoculated simultaneously or at different times, may differ in invasive rate, rate of gametocytogenesis, immunogenicity and immunosensitivity. Hellriegel [9] points out that in the absence of immunity, the more invasive clone will eliminate the less invasive one and in the presence of immunity, the two clones can coexist. This has been further studied by Mason and McKenzie [12] and Mason [13] on the effects of immune responses and drug intervention in mixed-species infections. Some recent models have exploited the discrete formulations $[15,17]$ 
limited to Plasmodium falciparum dynamics while other authors have studied age compartmental models $[5,7,8]$.

Gravenor [4] used Anderson [1] model without immunity to evaluate to what extent the consumption of red blood cells by $P$. falciparum can explain the control of the parasite density and the anemia observed during malaria therapy. They noted that the transient behaviour of their model does not correspond to the data. Gravenor [4] estimated multiplication factor of $P$. falciparum from prefebrile pretreatment data, assuming that the erythrocytic cycle of $P$. falciparum lasts for $48 \mathrm{~h}$. They obtained the value of reproductive rate (defined as the number of secondary infections which results from a single infection over the period of a case's infectivity).

Most mathematical models of within-host dynamics of asexual parasitaemia have been criticized [14] for their lack of biological realism. They take a general form of the prey-predator models, with the prey a population of Plasmodium and the predator a population of immune agents. These models usually considered as systems of ordinary differential equations that do not consider the time delay for the developmental period of the parasite as it replicates and differentiate into merozoites and the transmissible gametocyte stages. However, in the [3] model, a delay equation is used to investigate the sequestration time of a gametocyte in order to predict the conversion of gametocytaemia from asexual parasitaemia.

In the present paper, we propose a continuous model with age structure. The model considered here is an extension of Anderson [1] model in which we make a more realistic biological modification that incorporates an intracellular time delay within the red blood cell. We first formulate it as a system of partial differential equations that consists of four compartments: the healthy erythrocytes, parasitized erythrocytes, free merozoites and the immune cells for the blood-stage dynamics of a single species malaria infection. The model parameters are assumed to be ageand time-dependent. Under certain biologically realistic assumptions, the method of characteristics is employed to simplify the system of partial differential equations. These reduce to a system of coupled delay differential equations with a single time delay that represents time between infection of healthy erythrocytes and the emission of free merozoites into the blood stream on cellular level. Our goal is to study the qualitative behaviour of the parasite population growth during the blood stage of infection. Our model may readily be applied to infections that exhibit similar dynamics or even extended or modified to model the dynamics of interacting components.

The organization of this paper is as follows. In the next section, we formulate an age-structured model for the dynamics of the blood stage malaria. The method of characteristics is employed to reduce the system of partial differential equations to a system of delay differential equations. In Section 3 the system is discretized and then numerical simulation done. A discussion of the results is carried out in Section 4.

\section{Model Formulation}

We formulate a more descriptive model for interaction between the malaria parasites and immune system within a human host using a system of partial differential equations for the rate of change with respect to time and age of the density of 
malaria parasite populations and immune effectors. The uninfected red blood cells emerge from bone marrow at constant rate and are removed by phagocytosis [16] 120 days later due to loss of pliability of the cell membrane. The free merozoites invade the uninfected red blood cells within which they multiply and grow. The presence of the parasites induce specific immune response in the human host which grow in a parasite-dependent manner, and kills the parasite at a rate proportional to the product of the parasite and immune cells densities. Thus, we have an agestructured model since infection of the red blood cells. This puts into consideration of the period needed for replication and growth of the parasite within the infected red blood before it bursts to release 8-32 merozoites and gametocytes for the new generation of the next stage. The merozoites are removed from the replication cycle by the action of immune effectors. The gametocytes and parasitized red blood cells are captured, scavenged and removed by immune system cells in the spleen. Thus, time since infection of the cell is considered important in studying the dynamics of malaria within a human host. The interpretation of the variables and parameters used in the model is given in Subsection 2.2.

\subsection{Assumptions}

(i) Uninfected red blood cells are released from bone marrow at a constant rate and have a natural life expectancy

(ii) Red blood cells are infected at a rate proportional to their density

(iii) The death of infected cells due to maturation of schizonts is rapid compared to the above mentioned rates

(iv) The released merozoites either die or successfully infect new red blood cells

(v) There is a constant proportion of asexual parasites that convert to gametocytes within each cycle.

(vi) Infected red blood cells bursting at time $t$ were infected $a_{0}$ time units ago.

\subsection{Variables and Parameters}

The model definitions of variables and parameters are given as follows:
$X(t, a)$ : density of uninfected red blood cells of age $a$ at time $t$
$Y(t, a)$ : density of infected red blood cells of age $a$ at time $t$
$Z(t, a)$ : density of merozoites of age $a$ at time $t$
$G(t, a)$ : density of gametocytes of age $a$ at time $t$
$I(t, a)$ : density of specific immune cells of age $a$ at time $t$
$\mu_{x}: \quad$ natural death rate of uninfected red blood cells
$\mu_{y}:$ natural death rate of infected red blood cells
$\mu_{z}:$ natural death rate of merozoites
$\mu_{g}:$ natural death rate of gametocytes
$\mu_{i}$ : natural death rate of immune cells
$\mu_{1}(a)$ : age-dependent rate at which asexual forms differentiate into merozoites
$\mu_{2}(a)$ : age-dependent rate at which asexual forms differentiate into gametocytes
$\Lambda$ : rate at which red blood cells are produced by the bone marrow
$\kappa_{y}:$ the elimination rate of infected erythrocytes through the immune cells
$\kappa_{z}$ : elimination rate of merozoites through the immune cells
$\kappa_{g}$ : the elimination rate of gametocytes through the immune cells 
$\lambda_{y}:$ proliferation rate of the immune cells through infected red blood cells

$\lambda_{z}$ : proliferation rate of the immune cells through merozoites

$\lambda_{g}$ : proliferation rate of the immune cells through gametocytes

$\alpha$ : background rate of production of immune cells

$\beta$ : proliferation rate of immune cells

$\kappa: \quad$ contact rate between the uninfected red blood cells and the merozoites

$a_{0}$ : time interval between infection and burst of infected red blood cells

$r$ : number of merozoites released per dying infected red blood cell

\subsection{Equations of the Model}

The mathematical description of the change in the interacting stages of the parasites and immune response in the human host is described by the following system of partial differential equations, for $a$ and $t$ nonnegative.

$$
\begin{aligned}
\frac{\partial}{\partial t} X(t, a)+\frac{\partial}{\partial a} X(t, a)= & -\mu_{x} X(t, a)-k X(t, a) \int Z(t, b) d b \\
\frac{\partial}{\partial t} Y(t, a)+\frac{\partial}{\partial a} Y(t, a)= & -\mu_{y} Y(t, a)-\mu_{1}(a) Y(t, a)-\mu_{2}(a) Y(t, a) \\
& -k_{y} Y(t, a) \int I(t, b) d b \\
\frac{\partial}{\partial t} Z(t, a)+\frac{\partial}{\partial a} Z(t, a)= & -\mu_{z} Z(t, a)-k Z(t, a) \int X(t, b) d b \\
& -k_{z} Z(t, a) \int I(t, b) d b \\
\frac{\partial}{\partial t} G(t, a)+\frac{\partial}{\partial a} G(t, a)= & -\mu_{g} G(t, a)-k_{g} G(t, a) \int I(t, b) d b \\
\frac{\partial}{\partial t} I(t, a)+\frac{\partial}{\partial a} I(t, a)= & -\mu_{i} I(t, a)
\end{aligned}
$$

with the boundary conditions

$$
\begin{aligned}
X(t, 0)= & \Lambda, Y(t, 0)=k \int X(t, c) d c \int Z(t, b) d b \\
Z(t, 0)= & r \int \mu_{1}(b) Y(t, b) d b, \quad G(t, 0)=\int \mu_{2}(b) Y(t, b) d b \\
I(t, 0)= & f\left[\lambda_{y} \int Y(t, b) d b+\lambda_{z} \int Z(t, b) d b\right. \\
& \left.+\lambda_{g} \int G(t, b) d b\right]\left[\alpha+\beta \int I(t, b) d b\right], t>0
\end{aligned}
$$

\subsection{Reduction of the Model Equations to a System of Delay Equations}

The model presented above consists of a coupled system of partial differential equations that describe the age-structured populations of the blood-stage cycle of the parasite and immune cells. It is worthy noting that in our system of partial differential 
equations, the dependence of the right hand side is linear with age independent coefficients. We can eliminate the age dependence by integrating the equations of the system (1-5) with respect to the age variable, a from $a=0$ to $\infty$. The boundary conditions in Eq. 6 and the assumption $X(t, \infty)=Y(t, \infty)=Z(t, \infty)=G(t, \infty)=$ $I(t, \infty)=0$ are used. This is because from the biological reasoning point of view, we assume that no single individual of the population can survive to infinite age. The resulting equations are obtained as follows:

For healthy cells (uninfected red blood cells)

$$
\begin{aligned}
\partial_{t} \bar{X}(t) & =\frac{\partial}{\partial t} \int_{0}^{\infty} X(t, a) d a \\
& =\int_{0}^{\infty} \partial_{t} X(t, a) d a \\
& =-\int_{0}^{\infty} \partial_{a} X(t, a) d a-\mu_{x} \int_{0}^{\infty} X(t, a) d a-k \int_{0}^{\infty} X(t, a) d a \bar{Z}(t) \\
& =X(t, 0)-X(t, \infty)-\mu_{x} \int_{0}^{\infty} X(t, a) d a-k \int_{0}^{\infty} X(t, a) d a \bar{Z}(t) \\
& =\Lambda-\mu_{x} \bar{X}(t)-k \int_{0}^{\infty} X(t, a) d a \bar{Z}(t) \\
& =\Lambda-\mu_{x} \bar{X}(t)-k \bar{X}(t) \bar{Z}(t)
\end{aligned}
$$

The following argument is used to find the population densities

$$
\begin{aligned}
\partial_{s} X(t+s, s) & \leq-\mu_{x} X(t+s, s) \\
\Rightarrow X(t+s, s) & \leq X(t, 0) e^{-\mu_{x} s}, \quad t>0
\end{aligned}
$$

Thus, we have

$$
X(t, a) \leq X(t-a, 0) e^{-\mu_{x} a}, \quad t>a
$$

Similarly,

$$
X(t, a) \leq X(0, a-t) e^{-\mu_{x} t} \text { for, } t \leq a
$$

For the infected cells, we have

$$
\begin{aligned}
\partial_{t} Y(t, a)+\partial_{a} Y(t, a) & =-\mu_{y} Y(t, a)-\mu_{1}(a) Y(t, a)-\mu_{2}(a) Y(t, a)-k_{y} Y(t, a) \bar{I}(t) \\
Y(t, 0) & =k \bar{X}(t) \bar{Z}(t), t>0
\end{aligned}
$$

For the merozoites

$$
\partial_{t} \bar{Z}(t)=r \int_{0}^{\infty} \mu_{1}(a) Y(t, a) d a-\mu_{z} \bar{Z}(t)-k \bar{Z}(t) \bar{X}(t)-k_{z} \bar{Z}(t) \bar{I}(t)
$$

For the gametocytes

$$
\partial_{t} \bar{G}(t)=\int_{0}^{\infty} \mu_{2}(a) Y(t, a) d a-\mu_{g} \bar{G}(t)-k_{g} \bar{G}(t) \bar{I}(t) \quad t>0
$$


For antibodies

$$
\partial_{t} \bar{I}(t)=[\alpha+\beta \bar{I}(t)]\left[f\left(\lambda_{y} \bar{Y}(t)+\lambda_{z} \bar{Z}(t)+\lambda_{g} \bar{I}(t)\right)\right]-\mu_{i} \bar{I}(t) t>0
$$

It is important to note that in the dynamics of malaria infection within a host, in particular the P. falciparum, is categorized in terms of age classes based on parasite morphology stages within the infected red blood cell [8]. The erythrocyte phase has the first 12 or so hours after parasitic invasion of the cell the parasites are in the "young ring" stage while during the next 12 or so hours the parasites are in the "old ring" or "young trophozoites" development. These two stages of development of the parasite circulate freely in the blood stream and do not differentiate. The next 24 or so hours of the developmental stage is referred to as the "old ringt't' or "old trophozoites", until finally they become schizonts/segmenters. During this stage, the infected cells leave the circulating blood and sequester in the endothelium in the microvasculate. Thus infected cells in their stage of development tend to burst and release daughter parasites which invade new uninfected red blood cells when they are approximately $48 \mathrm{~h}$ old. We need to first state the assumptions on the age dependent model parameters $\mu_{1}(a)$ and $\mu_{2}(a)$ and assume that the infected red blood cells have a maximum age $a_{0}$ at which they begin to differentiate.

If

$$
\mu_{1}(a)=\left\{\begin{array}{l}
0, \text { for } a<a_{0} \\
M \cdot \mu_{1}, \text { for } a \geq a_{0}
\end{array}\right.
$$

and

$$
\mu_{2}(a)=\left\{\begin{array}{l}
0, \text { for } a<a_{0} \\
M \cdot \mu_{2}, \text { for } a \geq a_{0}
\end{array}\right.
$$

then we have the following

$$
\begin{aligned}
\frac{\partial}{\partial s}(Y(t+s, s)= & -\mu_{1}(s) Y(t+s, s)-\mu_{2}(s) Y(t+s, s)-\mu_{y} Y(t+s, s) \\
& -k_{y} Y(t+s, s) \bar{I}(t+s) \\
\partial s \ln (Y(t+s, s)= & -\mu_{y}-\mu_{1}(s)-\mu_{2}(s)-k_{y} \bar{I}(t+s)
\end{aligned}
$$

For $s<a_{0}$,

$$
Y(t+s, s)=Y(t, 0) \exp \left(-\mu_{y} s-k_{y} \int_{t}^{t+s} \bar{I}(\tau) d \tau\right)
$$

For $t>a$ and $a \leq a_{0}$,

$$
Y(t, a)=Y(t-a, 0) \exp \left(-\mu_{y} a-k_{y} \int_{t-a}^{t} \bar{I}(\tau) d \tau\right)
$$


and for $a>a_{0}$,

$$
\begin{aligned}
Y(t, a)= & Y(t-a, 0) \exp \left(\int_{0}^{a_{0}}-\left(\mu_{y}+\mu_{1}(s)+\mu_{2}(s)+k_{y} \bar{I}(t-a+s) d s\right)\right. \\
& \times \exp \left(\int_{a_{0}}^{a}-\left(\mu_{y}+M\left(\mu_{1}+\mu_{2}\right)+k_{y} \bar{I}(t-a+s) d s\right)\right. \\
= & Y(t-a, 0) \exp \left(-\mu_{y} a\right) \exp \left(-k_{y} \int_{0}^{a} \bar{I}(t-a+s) d s\right) \\
& \times \exp \left(-M\left(\mu_{1}+\mu_{2}\right)\left(a-a_{0}\right)\right)
\end{aligned}
$$

Thus, we establish how $\bar{Y}(t)$ appears in other equations as follows:

When $a \leq a_{0}$,

$$
\begin{aligned}
\bar{Y}(t) & =\int_{0}^{\infty} Y(t, a) d a \\
& =\int_{0}^{a_{0}} Y(t, a) d a \\
& =\int_{0}^{a_{0}} Y(t-a, 0) \exp \left(-\mu_{y} a\right) \exp \left(-k_{y} \int_{0}^{a} \bar{I}(t-a+s) d s\right) \\
& =\int_{0}^{a_{0}} k \bar{X}(t-a) \bar{Z}(t-a) \exp \left(-\mu_{y} a\right) \exp \left(-k_{y} \int_{0}^{a} \bar{I}(t-a+s) d s\right) d a
\end{aligned}
$$

When $a>a_{0}$ and $M$ goes to $\infty$,

$$
\begin{aligned}
\int_{0}^{\infty} \mu_{2}(a) Y(t, a) d a= & M \mu_{2} \int_{0}^{\infty} Y(t, a) d a \\
= & M \mu_{2} \int_{a_{0}}^{\infty} Y(t, a) d a \\
= & M \mu_{2} \int_{a_{0}}^{\infty} Y(t-a, 0)\left[\exp \left(-\mu_{y} a\right) \exp \left(-k_{y} \int_{0}^{a} \bar{I}(t-a+s) d s\right)\right. \\
& \left.\times \exp \left(-M\left(\mu_{1}+\mu_{2}\right)\left(a-a_{0}\right)\right)\right] d a \\
= & M \mu_{2} \int_{0}^{\infty} f(\sigma) \exp \left(-M\left(\mu_{1}+\mu_{2}\right) \sigma d \sigma\right)
\end{aligned}
$$

where

$$
f(\sigma)=Y\left(t-a_{0}-\sigma, 0\right) \exp \left(-\mu_{y}\left(a_{0}+\sigma\right)\right) \exp \left(-k_{y} \int_{0}^{a_{0}+\tau} \bar{I}\left(t-a_{0}-\tau+s\right) d s\right)
$$

and

$$
f(0)=Y\left(t-a_{0}, 0\right) \exp \left(-\mu_{y} a_{0}\right) \exp \left(-k_{y} \int_{0}^{a_{0}} \bar{I}\left(t-a_{0}+s\right) d s\right)
$$


Since the dynamics of the infected red blood cell is such that it reaches a time (of age) where it bursts, denote this cell age by $\delta$ in the interval $[0, \infty)$. Then Eq. 16 gives:

$$
\begin{array}{r}
M \int_{0}^{\delta} \exp \left(-M\left(\mu_{1}+\mu_{2}\right) s d s\right) \rightarrow \frac{1}{\mu_{1}+\mu_{2}} \text { as } M \rightarrow \infty \\
M \int_{\delta}^{\infty} \exp \left(-M\left(\mu_{1}+\mu_{2}\right) s d s\right) \rightarrow 0 \text { as } M \rightarrow \infty
\end{array}
$$

Thus,

$$
M \mu_{2} \int_{0}^{\infty} f(s) \exp \left(-M\left(\mu_{1}+\mu_{2}\right) s\right) d s=\frac{\mu_{2}}{\mu_{1}+\mu_{2}} \cdot f(0)
$$

Substituting Eq. 19 into Eq. 16 with the boundary conditions in Eq. 6 give

$$
\begin{aligned}
Z(t, 0)= & r \int \mu_{1}(b) Y(t, b) d b, \quad t>0 \\
= & \frac{r \mu_{1}}{\mu_{1}+\mu_{2}} k \bar{X}\left(t-a_{0}\right) \bar{Z}\left(t-a_{0}\right) \\
& \times \exp \left(-\mu_{y} a_{0}\right) \exp \left(-k_{y} \int_{0}^{a_{0}} \bar{I}\left(t-a_{0}+s\right) d s\right)
\end{aligned}
$$

and

$$
\begin{aligned}
G(t, 0)= & \int \mu_{2}(b) Y(t, b) d b, \quad t>0 \\
= & \frac{\mu_{2}}{\mu_{1}+\mu_{2}} k \bar{X}\left(t-a_{0}\right) \bar{Z}\left(t-a_{0}\right) \\
& \times \exp \left(-\mu_{y} a_{0}\right) \exp \left(-k_{y} \int_{0}^{a_{0}} \bar{I}\left(t-a_{0}+s\right) d s\right)
\end{aligned}
$$

The coupled system of partial differential Eqs. 1-5 has been reduced to system Eqs. 22-25 of delay differential equations that incorporates a realistic physiology associated with the intra cellular time delay $a_{0}$ that accounts for the time required for the parasites to replicate and grow within parasitized red blood cells until they release free merozoites into the blood stream when they burst. The equation for the infected red blood cells now appear as expressions for birth terms in Eqs. 20 and 21 for the merozoites and gametocytes respectively. The density of infected red blood cells only in the equations in terms of their elimination rate through the immune cells. Thus, 
we have the following reduced system of delay equations for the population densities of uninfected red blood cells, merozoites, gametocytes and immune cells.

$$
\begin{aligned}
\partial_{t} \bar{X}(t)= & \Lambda-\mu_{x} \bar{X}(t)-k \bar{X}(t) \bar{Z}(t) \\
\partial_{t} \bar{Z}(t)= & \frac{r \mu_{1}}{\mu_{1}+\mu_{2}} k \bar{X}\left(t-a_{0}\right) \bar{Z}\left(t-a_{0}\right) \exp \left(-\mu_{y} a_{0}\right) \exp \left(-k_{y} \int_{0}^{a_{0}} \bar{I}\left(t-a_{0}+s\right) d s\right) \\
& -\mu_{z} \bar{Z}(t)-k \bar{X}(t) \bar{Z}(t)-k_{z} \bar{Z}(t) \bar{I}(t), \quad t>a_{0} \\
\partial_{t} \bar{G}(t)= & \frac{\mu_{2}}{\mu_{1}+\mu_{2}} k \bar{X}\left(t-a_{0}\right) \bar{Z}\left(t-a_{0}\right) \exp \left(-\mu_{y} a_{0}\right) \exp \left(-k_{y} \int_{0}^{a_{0}} \bar{I}\left(t-a_{0}+s\right) d s\right) \\
& -\mu_{g} \bar{G}(t)-k_{g} \bar{G}(t) \bar{I}(t), \quad t>a_{0} \\
\partial_{t} \bar{I}(t)= & {[\alpha+\beta \bar{I}(t)] f\left[\lambda_{y} \int_{0}^{a_{0}} k \bar{X}(t-a) \bar{Z}(t-a) \exp \left(-\mu_{y} a\right)\right.} \\
& \left.\times \exp \left(-k_{y} \int_{0}^{a} \bar{I}(t-a+s) d s\right) d a\right] \\
& \left.+\lambda_{z} \bar{Z}(t)+\lambda_{g} \bar{G}(t)\right]-\mu_{i} I(t), \quad t>a_{0}
\end{aligned}
$$

In some situations, the density of infected red blood cells depends on the density of the red blood cells initially infected. Assuming that the initial age-structure of infected red blood cells satisfies $Y(0, a)=Y_{0}(a)$. The method of characteristics is applied to find the solution to the partial equation for infected red blood cells as follows:

For the case $t<a$, and $Y(0, a)=Y_{0}(a)$

$$
\begin{aligned}
\frac{\partial}{\partial s}(Y(t+s, a+s)= & -\mu_{1}(a+s) Y(t+s, a+s)-\mu_{2}(a+s) Y(t+s, a+s) \\
& -\mu_{y} Y(t+s, a+s)-k_{y} Y(t+s, a+s) \bar{I}(t+s) \\
\partial s \ln (Y(t+s, a+s)= & -\mu_{y}-\mu_{1}(a+s)-\mu_{2}(a+s)-k_{y} \bar{I}(t+s)
\end{aligned}
$$

For $s+a<a_{0}$,

$$
Y(t+s, a+s)=Y(t, a) \exp \left(-\mu_{y} s-k_{y} \int_{0}^{s} \bar{I}(t+\tau) d \tau\right)
$$

For $t<a<a_{0}$,

$$
\begin{aligned}
Y(t, a) & =Y(0, a-t) \exp \left(-\mu_{y} t-k_{y} \int_{0}^{t} \bar{I}(\tau) d \tau\right) \\
& =Y_{0}(a-t) \exp \left(-\mu_{y} t\right) \exp \left(-k_{y} \int_{0}^{t} \bar{I}(\tau) d \tau\right)
\end{aligned}
$$


and for $t<a, a>a_{0}$,

$$
\begin{aligned}
Y(t, a)= & Y(0, a-t) \exp \left(-\mu_{y} t\right) \exp \left(-k_{y} \int_{0}^{t} \bar{I}(\tau) d \tau\right) \\
& \times \exp \left(-M\left(\mu_{1}+\mu_{2}\right) \min \left(a-a_{0}, t\right)\right) \\
= & Y_{0}(a-t) \exp \left(-\mu_{y} t\right) \exp \left(-k_{y} \int_{0}^{t} \bar{I}(\tau) d \tau\right) \\
& \times \exp \left(-M\left(\mu_{1}+\mu_{2}\right) \min \left(a-a_{0}, t\right)\right)
\end{aligned}
$$

$\bar{Y}(t)$ appears in other equations in the following ways that are considered in two cases, i.e. $t<a_{0}$ and $t>a_{0}$ :

For $t<a_{0}, a<t \Rightarrow a<a_{0}$

$$
\begin{aligned}
\bar{Y}(t)= & \int_{0}^{\infty} Y(t, a) d a \\
= & k \int_{0}^{t}\left(\bar{X}(t-\tilde{a}) \bar{Z}(t-\tilde{a}) \exp \left(-\mu_{y} \tilde{a}\right) \exp \left(-k_{y} \int_{t-\tilde{a}}^{t} \bar{I}(\tau) d \tau\right)\right. \\
& +\int_{t}^{a_{0}} Y(0, a-t) \exp \left(-\mu_{y} t\right) \exp \left(-k_{y} \int_{0}^{t} \bar{I}(\tau) d \tau\right) \\
& +\int_{a_{0}}^{\infty} Y(0, a-t) \exp \left(-\mu_{y} t\right) \exp \left(-k_{y} \int_{0}^{t} \bar{I}(\tau) d \tau\right) \\
& \times \exp \left(-M\left(\mu_{1}+\mu_{2}\right) \min \left(a-a_{0}, t\right)\right)
\end{aligned}
$$

We calculate $Y\left(t, a_{0}\right)$ along the characteristics as follows:

For $t<a_{0}$, the characteristic curve passes through $\left(t, a_{0}\right)$ and $(0, c)$, where $c$ is the intercept on $a$-axis given by $c=a_{0}-t$. This gives

$$
Y\left(t, a_{0}\right)=Y(0, a-t) \exp \left(-\mu_{y} t\right) \exp \left(-k_{y} \int_{0}^{t} \bar{I}(\tau) d \tau\right)
$$

For $t>a_{0}$, the characteristic curve passes through $\left(t, a_{0}\right)$ and $\left(c^{\prime}, 0\right)$, that satisfies $c^{\prime}=t-a_{0}$, where $c^{\prime}$ is the intercept on $t$-axis. So, substituting for $\left(t-a_{0}, 0\right)$ in the expression for the birth term $Y(t, 0)=k \bar{X}(t) \bar{Z}(t)$ gives $Y\left(t-a_{0}, 0\right)=k \bar{X}(t-$ $\left.a_{0}\right) \bar{Z}\left(t-a_{0}\right)$ and hence

$$
Y\left(t, a_{0}\right)=k\left(\bar{X}\left(t-a_{0}\right) \bar{Z}\left(t-a_{0}\right) \exp \left(-\mu_{y} a_{0}\right) \exp \left(-k_{y} \int_{t-a_{0}}^{t} \bar{I}(\tau) d \tau\right)\right.
$$

Thus, we have the expression

$$
Y\left(t, a_{0}\right)=\left\{\begin{array}{l}
Y(0, a-t) \exp \left(-\mu_{y} t\right) \exp \left(-k_{y} \int_{0}^{t} \bar{I}(\tau) d \tau\right), \text { for } t<a_{0} \\
k\left(\bar{X}\left(t-a_{0}\right) \bar{Z}\left(t-a_{0}\right) \exp \left(-\mu_{y} a_{0}\right) \exp \left(-k_{y} \int_{t-a_{0}}^{t} \bar{I}(\tau) d \tau\right), \text { for } t>a_{0}\right.
\end{array}\right.
$$


For values larger than $a_{0}$, we continue along the characteristics and calculate $Y\left(t, a_{0}+\tilde{a}\right)$. For $t<\tilde{a}$, the characteristic curve passes through $\left(t, a_{0}+\tilde{a}\right)$ and $(0, c)$, where $c$ is the intercept on the $a$-axis given by $c=a_{0}+\tilde{a}-t$. This gives

$$
Y\left(t, a_{0}+\tilde{a}\right)=Y\left(0, a_{0}+\tilde{a}-t\right) \exp \left(-\left(\mu_{y}+M\left(\mu_{1}+\mu_{2}\right) t\right) \exp \left(-k_{y} \int_{0}^{t} \bar{I}(\tau) d \tau\right)\right.
$$

For $t>\tilde{a}$, the characteristic curve passes through $\left(t, a_{0}+\tilde{a}\right)$ and $\left(c, a_{0}\right)$, where $c$ is the intercept on the $a_{0}$-axis given by $c=t-\tilde{a}$. This gives

$$
Y\left(t, a_{0}+\tilde{a}\right)=Y\left(t-\tilde{a}, a_{0}\right) \exp \left(-\left(\mu_{y}+M\left(\mu_{1}+\mu_{2}\right) t\right) \exp \left(-k_{y} \int_{t-\tilde{a}}^{t} \bar{I}(\tau) d \tau\right)\right.
$$

Thus, we have

$$
Y\left(t, a_{0}+\tilde{a}\right)=\left\{\begin{array}{l}
Y\left(0, a_{0}+\tilde{a}-t\right) \exp \left(-\left(\mu_{y}+M\left(\mu_{1}+\mu_{2}\right) t\right) \exp \left(-k_{y} \int_{0}^{t} \bar{I}(\tau) d \tau\right),\right. \\
\text { for } t<\tilde{a} \\
Y\left(t-\tilde{a}, a_{0}\right) \exp \left(-\left(\mu_{y}+M\left(\mu_{1}+\mu_{2}\right) t\right) \exp \left(-k_{y} \int_{t-\tilde{a}}^{t} \bar{I}(\tau) d \tau\right),\right. \\
\text { for } t>\tilde{a}
\end{array}\right.
$$

We note that it is not easy to explicitly solve our system of equations as delay equations are notoriously difficult to analyze. However, we can still investigate its qualitative behaviour by approximating definite integrals by the trapezium rule and then carrying out discretization. This is done in the following section.

\section{Numerical Analysis of the Model}

In order to obtain numerical solution for the model, the time variable $t \geq 0$ is discretized at the points $t_{n}=t_{0}+n h(n=0,1,2, \ldots)$, where $h=t_{n+1}-t_{n}$ is a constant step-size. The finite-forward difference numerical methods that are firstorder approximations of the Euler method are used and the right hand side functions approximated in ways that give implicit methods. Implicit Euler is numerically stable for all step sizes. The solutions of our system at the grid point $t_{n}$ are $\bar{X}\left(t_{n}\right), \bar{Z}\left(t_{n}\right), \bar{G}\left(t_{n}\right), \bar{I}\left(t_{n}\right)$. We denote the solutions of numerical method at the point $t_{n}$ by $\bar{X}^{n}, \bar{Z}^{n}, \bar{G}^{n}, \bar{I}^{n}$ respectively.

$$
\begin{aligned}
\partial_{t} \bar{X}(t) & =\frac{\bar{X}(t+h)-\bar{X}(t)}{h} \\
\partial_{t} \bar{Z}(t) & =\frac{\bar{Z}(t+h)-\bar{Z}(t)}{h} \\
\partial_{t} \bar{G}(t) & =\frac{\bar{G}(t+h)-\bar{G}(t)}{h} \\
\partial_{t} \bar{I}(t) & =\frac{\bar{I}(t+h)-\bar{I}(t)}{h}
\end{aligned}
$$

Let

$$
N(t)=\int_{0}^{a_{0}} \bar{I}\left(t-a_{0}+s\right) d s=\int_{t-a_{0}}^{t} \bar{I}(\tau) d \tau \text { and } h=\frac{a_{0}}{n}
$$


where $h$ is the step size and $n$ is an integer. Then from the trapezoidal approximations, we have

$$
\begin{aligned}
N(t) & =\int_{t-a_{0}}^{t} \bar{I}(\tau) d \tau=h\left[\frac{1}{2} \bar{I}\left(t-a_{0}\right)+\bar{I}\left(t+h-a_{0}\right)+\ldots+\bar{I}(t-h)+\frac{1}{2} \bar{I}(t)\right] \\
N(t+h) & =\int_{t+h-a_{0}}^{t+h} \bar{I}(\tau) d \tau=h\left[\frac{1}{2} \bar{I}\left(t+h-a_{0}\right)+\ldots+\bar{I}(t-h)+\bar{I}(t)+\frac{1}{2} \bar{I}(t+h)\right] \\
& =\int_{t-a_{0}}^{t} \bar{I}(\tau) d \tau+\frac{h}{2}\left[\bar{I}(t+h)+\bar{I}(t)-\bar{I}\left(t-a_{0}\right)-\bar{I}\left(t+h-a_{0}\right)\right]
\end{aligned}
$$

from which we have

$$
N(t+h)=N(t)+\frac{h}{2}\left[\bar{I}(t+h)+\bar{I}(t)-\bar{I}\left(t-a_{0}\right)-\bar{I}\left(t+h-a_{0}\right)\right]
$$

Letting

$$
\begin{aligned}
M(t) & =\int_{0}^{a_{0}}\left[\bar{X}(t-a) \bar{Z}(t-a) \exp \left(-\mu_{y} a\right) \exp \left(-k_{y} \int_{0}^{a} \bar{I}(t-a+s) d s\right)\right] d a \\
& =\int_{0}^{a_{0}}\left[\bar{X}(t-a) \bar{Z}(t-a) \exp \left(-\mu_{y} a\right) \exp \left(-k_{y} \int_{t-a}^{t} \bar{I}(\tau) d \tau\right)\right] d a \\
& =\int_{t-a_{0}}^{t}\left[\bar{X}(\sigma) \bar{Z}(\sigma) \exp \left(-\mu_{y}(t-\sigma)\right) \exp \left(-k_{y} \int_{t-a}^{t} \bar{I}(\tau) d \tau\right)\right] d \sigma
\end{aligned}
$$

and

$$
\begin{aligned}
\int_{t-a_{0}}^{t} & {\left[\bar{X}(\sigma) \bar{Z}(\sigma) \exp \left(-\mu_{y}(t+h-\sigma)\right) \exp \left(-k_{y} \int_{t-a}^{t+h} \bar{I}(\tau) d \tau\right] d \sigma\right.} \\
= & \int_{t-a_{0}}^{t} \bar{X}(\sigma) \bar{Z}(\sigma) \exp \left(-\mu_{y} h\right) \exp \left(-\mu_{y}(t-\sigma)\right) \\
& \times \exp \left(-k_{y} \int_{t-a}^{t} \bar{I}(\tau) d \tau\right) d \sigma \exp \left(-k_{y} h \bar{I}(t)\right)=M(t) \exp \left(-h\left(\mu_{y}+k_{y} \bar{I}(t)\right)\right)
\end{aligned}
$$

then

$$
\begin{aligned}
\int_{t+h-a_{0}}^{t+h} & {\left[\bar{X}(s) \bar{Z}(s) \exp \left(-\mu_{y}(t+h-s) \exp \left(-k_{y} \int_{s}^{t+h} \bar{I}(\tau) d \tau\right)\right] d s\right.} \\
= & M(t) \exp \left(-h\left(\mu_{y}+k_{y} \bar{I}(t)\right)\right)+\frac{h}{2}[\bar{X}(t+h) \bar{Z}(t+h)+\bar{X}(t) \bar{Z}(t)] \\
- & \frac{h}{2}\left[\bar{X}\left(t+h-a_{0}\right) \bar{Z}\left(t+h-a_{0}\right)\right. \\
& \left.+\bar{X}\left(t-a_{0}\right) \bar{Z}\left(t-a_{0}\right) \exp \left(-\mu_{y} a_{0}-k_{y} \int_{t-a_{0}}^{t} \bar{I}(\tau) d \tau\right)\right]
\end{aligned}
$$


so that

$$
\begin{aligned}
M(t+h)= & M(t) \exp \left(-h\left(\mu_{y}+k_{y} \bar{I}(t)\right)\right)+\frac{h}{2}[\bar{X}(t+h) \bar{Z}(t+h)+\bar{X}(t) \bar{Z}(t)] \\
& -\frac{h}{2}\left[\bar{X}\left(t+h-a_{0}\right) \bar{Z}\left(t+h-a_{0}\right)+\bar{X}\left(t-a_{0}\right) \bar{Z}\left(t-a_{0}\right)\right] \\
& \times \exp \left(-\mu_{y} a_{0}-k_{y} \int_{t-a_{0}}^{t} \bar{I}(s) d s\right)
\end{aligned}
$$

Substituting the terms in expression Eq. 33 together with the trapezoidal approximations Eqs. 34-36 and 37 for the integral terms into the Eqs. 22-25 on simplification give the following expressions:

$$
\begin{aligned}
& \bar{X}(t+h)=\frac{\bar{X}(t)+\Lambda h}{1+h\left(\mu_{x}+k \bar{Z}(t)\right)} \\
& \bar{Z}(t+h)=\frac{\bar{Z}(t)+\frac{h r \mu_{1}}{\mu_{1}+\mu_{2}} k \bar{X}\left(t-a_{0}\right) \bar{Z}\left(t-a_{0}\right) \exp \left(-\mu_{y} a_{0}\right) \exp \left(-k_{y} N(t)\right)}{1+h\left(\mu_{z}+k \bar{X}(t)+k_{z} \bar{I}(t)\right)} \\
& \bar{G}(t+h)=\frac{\bar{G}(t)+\frac{h \mu_{2}}{\mu_{1}+\mu_{2}} k \bar{X}\left(t-a_{0}\right) \bar{Z}\left(t-a_{0}\right) \exp \left(-\mu_{y} a_{0}\right) \exp \left(-k_{y} N(t)\right.}{1+h\left(\mu_{g}+k_{g} \bar{I}(t)\right)} \\
& \bar{I}(t+h)=\frac{\left[\bar{I}(t)+h\left(\alpha \lambda_{y} k M(t)+\lambda_{z} \bar{Z}(t)+\lambda_{g} \bar{G}(t)\right]\right.}{1+h\left(\mu_{i}-k \lambda_{y} \beta M(t)\right)}
\end{aligned}
$$

where

$$
\begin{aligned}
N(t+h)= & N(t)+\frac{h}{2}\left[\bar{I}(t+h)+\bar{I}(t)-\bar{I}\left(t+h-a_{0}\right)-\bar{I}\left(t-a_{0}\right)\right] \\
M(t+h)= & M(t) \exp \left(-h\left(\mu_{y}+k_{y} \bar{I}(t)\right)\right) \\
& +\frac{h}{2}[\bar{X}(t+h) \bar{Z}(t+h)+\bar{X}(t) \bar{Z}(t)] \\
& -\frac{h}{2}\left[\bar{X}\left(t+h-a_{0}\right) \bar{Z}\left(t+h-a_{0}\right)+\bar{X}\left(t-a_{0}\right) \bar{Z}\left(t-a_{0}\right)\right] \\
& \times \exp \left(-\mu_{y} a_{0}-k_{y} N(t)\right)
\end{aligned}
$$

With the initial set of data and parameter values, we carry out numerical simulations of Eqs. 38-41. We can always compute $\bar{N}(t+h)$ and $\bar{M}(t+h)$ from Eqs. 42 and 43. Estimates of the relevant model parameters for the dynamics of the infection that are used to numerically examine the behaviour of the model are given in Table 1. It should be pointed out that our discretized system satisfies the positivity property in that for any positive initial data, our system will give positive solutions which are located in the feasible region.

\section{Discussion}

A mathematical model for the within-host dynamics of malaria parasites was formulated. Our main focus was the erythrocytic blood-stage asexual cycle of $P$. falciparum 
Table 1 Parameter estimates for the model of malaria parasites within a human host

\begin{tabular}{|c|c|c|c|}
\hline Symbol & Biological meaning & Value & Ref. \\
\hline$\Lambda$ & Rate of red blood cells production & $2.5 \times 10^{8} /$ cells $/ \mathrm{ml} /$ day & {$[2,10]$} \\
\hline$\mu_{x}$ & Natural death rate of un infected red blood cells & $0.0083 /$ day & {$[1,16]$} \\
\hline$\kappa$ & Infection rate of red blood cells by merozoites & $2 \times 10^{-9} /$ day & [2] \\
\hline$a_{0}$ & Life-span of infected red blood cells & 2 days & {$[3]$} \\
\hline$\mu_{y}$ & Natural death rate of infected red blood cells & $0.025 /$ day & [2] \\
\hline$\mu_{z}$ & Natural death rate of free merozoites & 48/day & [2] \\
\hline$\mu_{g}$ & Natural death rate of gametocytes & $0.06 /$ day & [9] \\
\hline$\mu_{i}$ & Natural death rate of immune cells & $0.05 /$ day & {$[1]$} \\
\hline$\mu_{1}$ & Differentiation rate of asexual form into merozoites & $0.5 /$ day & {$[1,10]$} \\
\hline$\mu_{2}$ & Differentiation rate of asexual form into gametocytes & $0.04 /$ day & {$[12]$} \\
\hline$r$ & Merozoites released per dying infected red blood cell & 16 & {$[1,3]$} \\
\hline$k_{z}$ & Elimination rate of merozoites through immune cells & $10^{-8} /$ day & [2] \\
\hline$k_{g}$ & $\begin{array}{l}\text { Elimination rate of gametocytes through } \\
\text { immune cells }\end{array}$ & $10^{-8} /$ day & [2] \\
\hline$\lambda_{y}$ & $\begin{array}{l}\text { Proliferation rate of immune cells in response to } \\
\text { infected red blood cells }\end{array}$ & $0.0001-1,000 /$ day & {$[2,12]$} \\
\hline$\lambda_{z}$ & $\begin{array}{l}\text { Proliferation rate of immune cells in response } \\
\text { to merozoites }\end{array}$ & $0.0001-1,000 /$ day & {$[2,12]$} \\
\hline$\lambda_{g}$ & $\begin{array}{l}\text { Proliferation rate of immune cells in response } \\
\text { to gametocytes }\end{array}$ & $0.0001-1,000 /$ day & {$[2,12]$} \\
\hline$\alpha$ & Background replication of immune cells & $2 \times 10^{-8} /$ day & [9] \\
\hline$\beta$ & Proliferation rate of non-specific immune cells & $0.0001-1,000 /$ day & {$[12]$} \\
\hline
\end{tabular}

that is associated with clinical symptoms and the related mortality. The model extends the Anderson [1] model to include an intracellular time delay within the red blood cell. The model was formulated in terms of partial differential equations so as to capture some important features that would augment other within-host models and make them more biologically realistic. The system was integrated along characteristics and using simplifying assumptions, it was reduced to a coupled system of delay differential equations. One of the assumptions made was that there is a finite time delay between infection of the red blood cell by the merozoite and release of the merozoites and gametocytes for the next generation. Thus, there is no infinite age of the infected red blood cell and parasite. We introduced a function $M$ which is dependent on the infected cell life-span. In the early stages $\left(a<a_{0}\right)$ of development, the parasites replicate and grow within the infected cells. The infected cells are moving freely in the peripheral blood and there is no bursting of the infected cells $\left(\mu_{i}(a)=0, i=1,2\right)$. However, towards the last phase of the parasite develop $\left(a \geq a_{0}\right)$, the infected red blood cells sequester in the vasculate organ and burst at a fast rate $\left(\mu_{i}(a)=M \mu_{i}, i=1,2\right)$. Thus, we assumed that there is a maximum age for the infected red blood cell, $a_{0}$. The variable for the infected red blood cells now appears in the variables for the merozoites and gametocytes hence reducing the burden of solving the original system of five partial differential equations. The delay effect incorporated is a more realistic physiology associated with a finite intracellular time delay between infection of the red blood cell and the emission of malaria parasites into blood stream. 


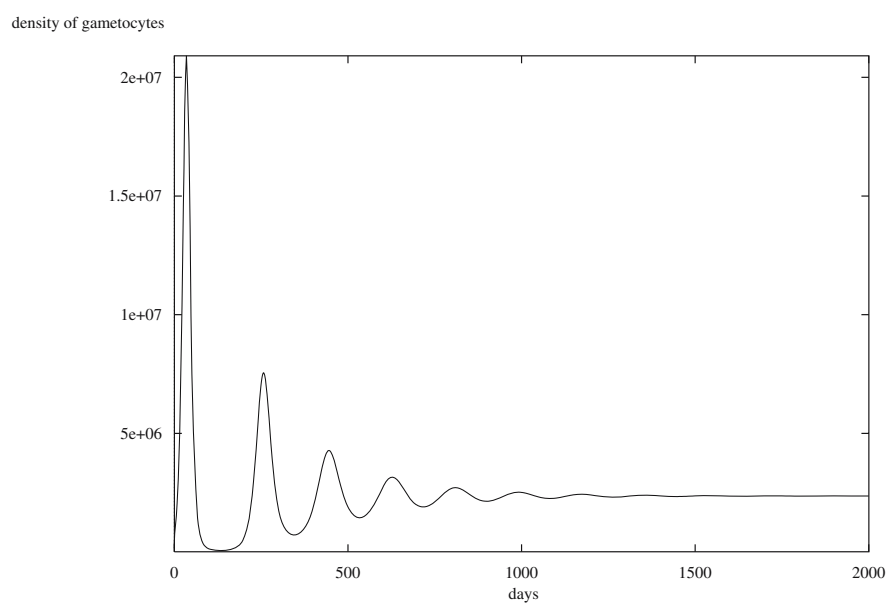

density of merozoites

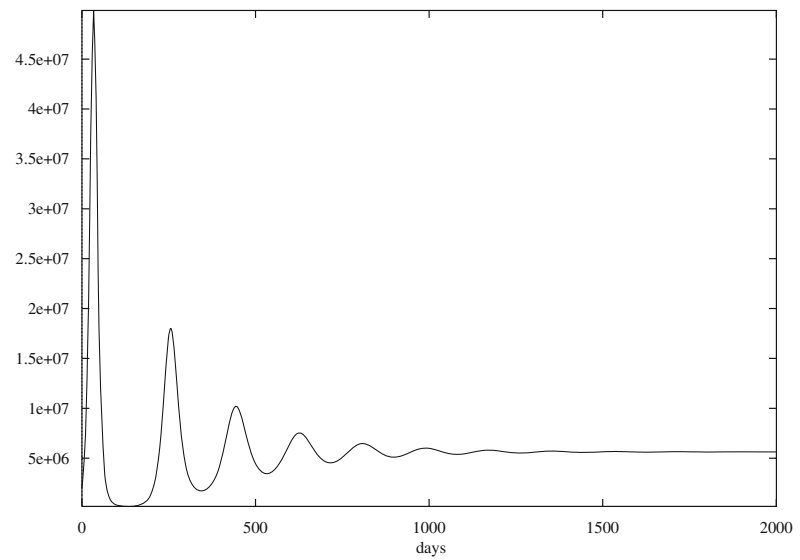

density of uninfected rbc

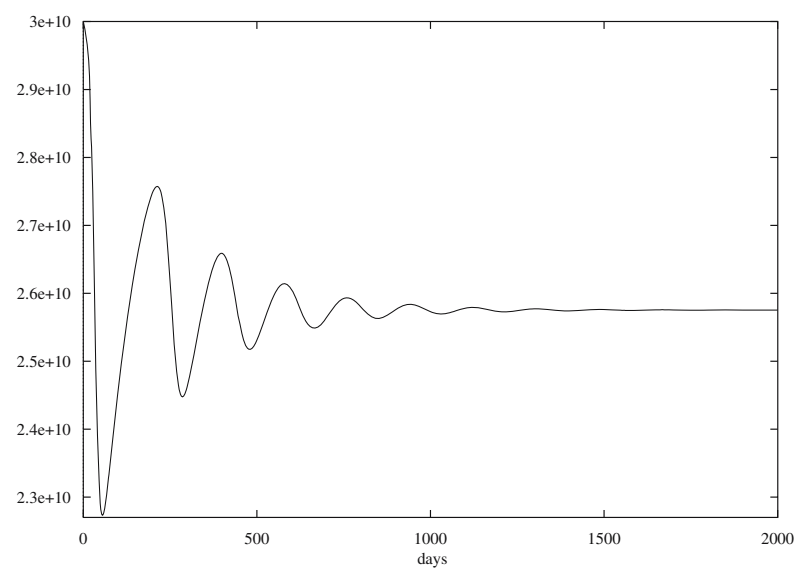

Fig. 1 The oscillation of the uninfected red blood cells, merozoites and gametocytes converge to a steady state when $r=16, a_{0}=48$ hours 


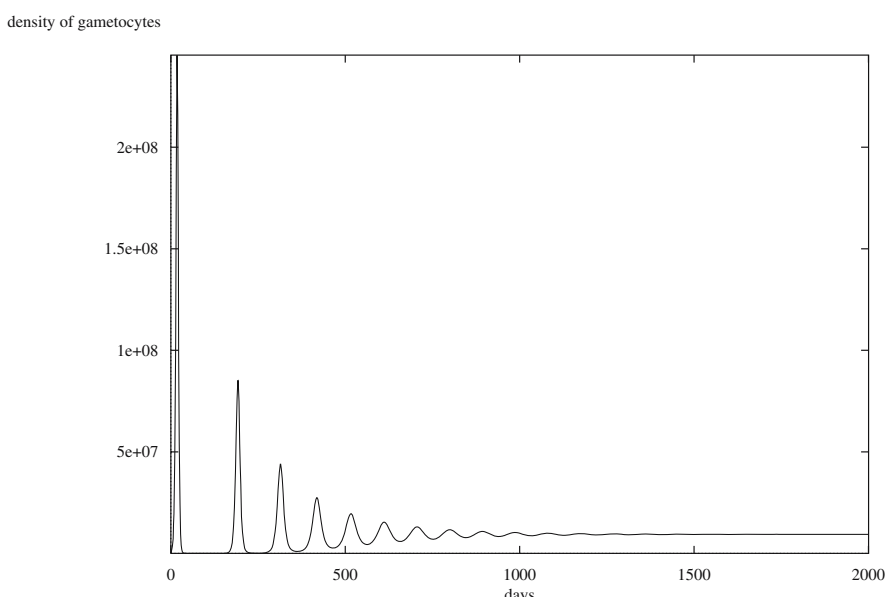

density of merozoites

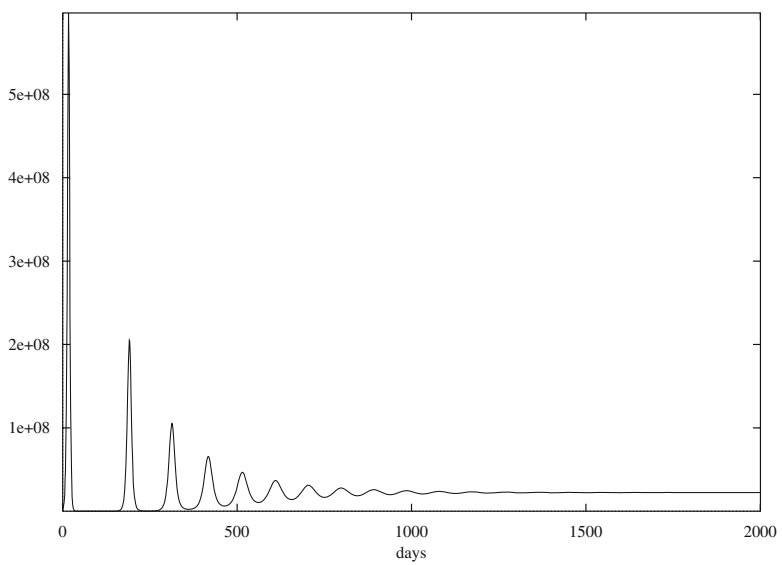

density of uninfected rbc

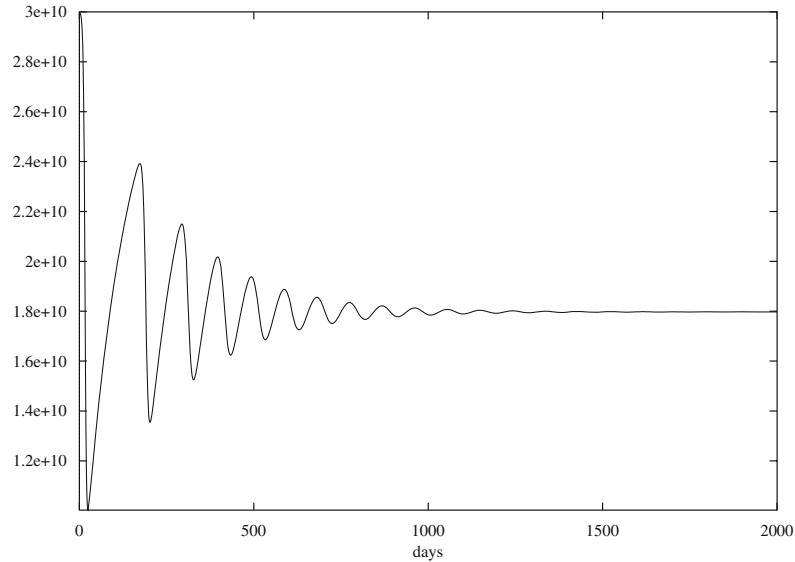

Fig. 2 The oscillation of the uninfected red blood cells, merozoites and gametocytes converge to a steady state when $r=12, a=24<a_{0}$ hours 
Although we had an idealized system of delay differential equations, it was not easy to explicity solve it. However, we could still investigate the qualitative properties of the model to demonstrate behaviour of the steady states. We descritized the system using finite forward difference for the numerical analysis. Simulations were carried out using the parameters in Table 1 to investigate the within-host dynamics of malaria infection over time.

The model exhibits oscillatory behaviour. It is observed that after the release of the parasites into the blood stream and the eventual invasion of the red blood cells, there is a sharp fall in the level of uninfected red blood density and a corresponding rise in the level of the density of merozoites and gametocytes (see Figs. 1 and 2). The density of the merozoites and gametocytes falls when the density of uninfected red blood cells available for invasion falls below the threshold level necessary to sustain the parasite growth. However, when sufficient healthy (uninfected) red blood cells are available for invasion, there is again a renewed rise in the level of the density of merozoites and gametocytes, which eventually results in the decline in the density of uninfected red blood cells.

Our model results reveal that the density of the merozoites and gametocytes released by the infected red blood cells rise and then fall via damped oscillations to a stable steady state. This is a more realistic result, noting that when the merozoites are released into the blood stream at the onset of the infection, the immune response sets in to fight it off until it is brought under control to a stable steady state. This is further established by simulations when the parameter values of red blood cell and release of the new steady state for the given parameter values.

The results obtained in this paper show that the steady state of the model of the within-host dynamics of malaria is stable with transient oscillations in the components of the system. Our model adds more considerable details such as replication and growth period within the infected cell that make the model more biologically realistic.

Acknowledgements We gratefully acknowledge the support from Deutscher Akademischer Austauschdienst (DAAD). This research was partly done when Julius Tumwiine was at the Max Planck Institute for Mathematics in the Sciences and University of Leipzig, Germany on DAAD scholarship student exchange programme as a PhD student.

\section{References}

1. Anderson, R.M., May, R.M., Gupta, S.: Non-linear phenomena in host-parasite interactions. Parasitology 99, S59-S79 (1989)

2. Austin, D.J., White, N.J., Anderson, R.M.: The dynamics of drug action on the within-host population growth of infectious agents: melding pharmacokinetics with pathogen population dynamics. J. Theor. Biol. 194, 313-339 (1998)

3. Diebner, H.H., Eichner, M., Molineaux, L., Collins, W.E., Jeffrey, G.M., Dietz, K.: Modelling the transition of asexual blood stages of plasmodium falciparum to gametocytes. J. Theor. Biol. 202, 113-127 (2000)

4. Gravenor, M.B., McLean, A.R., Kwiatkowski, D.: The regulation of malaria parasitaemia: parameter estimates for a population model. Parasitology 110, 115-122 (1995)

5. Gravenor, M.B., Kwiatkowski, D.: An analysis of temperature effects of fever on the intra-host population dynamics of Plasmodium falciparum. Parasitology 117, 97-105 (1998)

6. Gravenor, M.B., Boele Van Hensbroek, M., Kwiatkowski, D.: Estimating sequestered parasite population dynamics in celebral malaria. Proc. Natl. Acad. Sci. U. S. A. 95, 7620-7624 (1998)

Springer 
7. Gravenor, M.B., Lloyd, A.L.: Reply to: models for intra-host dynamics of malaria revisited: errors in some basic models lead to large overestimates of growth rates. Parasitology 117, 409-410 (1998)

8. Gravenor, M.B., Lloyd, A.L., Kremsner, P.G., Missinou, A., English, M., Marsh, K., Kwiatkowski, D.: A model for estimating total parasite load in falciparum malaria patients. J. Theor. Biol. 217, 137-148 (2002)

9. Hellriegel, B.: Modelling the immune response to malaria with ecological concepts: short-term behaviour against long-term equilibrium. Proc. R. Soc. Lond. 250, 249-256 (1992)

10. Hetzel, C., Anderson, R.M.: The within-host cellular dynamics of blood stage malaria. Theoretical and experimental studies. Parasitology 113, 25-38 (1996)

11. Hoshen, M.B., Heinrich, R., Stein, W.D., Ginsburg, H.: Mathematical modelling of the withinhost dynamics of Plasmodium falciparum. Parasitology 121, 227-235 (2000)

12. Mason, D.P., McKenzie, F.E.: Blood-stage dynamics and clinical implications of mixed Plasmodium vivax-Plasmodium falciparum infections. Am. J. Trop. Med. Hyg. 61(3), 367-374 (1999)

13. Mason, D.P., McKenzie, F.E., Bossert, W.H.: The Blood-stage dynamics of mixed implications of mixed Plasmodium malariae-Plasmodium falciparum infections. J. Theor. Biol. 198, 549-566 (1999)

14. Molineaux, L., Dietz, K.: Review of intra-host models of malaria. Parassitologia 41, 221-231 (1999)

15. Molineaux, L., Diebner, H.H., Eichner, M., Collins, W.E., Jeffery, G.M., Dietz, K.: Plasmodium falciparum parasitaemia described by a new mathematical model. Parasitology 122, 379-391 (2001)

16. Rapaport, S.I.: Introduction to Hematology. Lippincott, Philadelphia (1987)

17. White, N.J., Chapman, D., Watt, G.: The effects of multiplication and synchronicity on the vascular distribution of parasites in falciparum malaria. Trans. R. Soc. Trop. Med. Hyg. 86, 590-597 (1992) 